\title{
Efeito da Interação Reprodutor x Rebanho sobre as Produções de Leite e de Gordura na Raça Pardo-Suíça
}

\author{
Mirella Leme Franco Geraldini Sirol ${ }^{1}$, Ricardo Frederico Euclydes ${ }^{2}$, Robledo de Almeida Torres ${ }^{3}$, \\ Paulo Sávio Lopes ${ }^{3}$, Carmen Silva Pereira ${ }^{4}$, Cláudio Vieira Araújo ${ }^{5}$, Francisco Palma Rennó ${ }^{2}$
}

\begin{abstract}
RESUMO - Dados de 4.959 lactações de 2.414 vacas da raça Pardo-Suíça, filhas de 70 reprodutores, distribuídos em 51 rebanhos, foram utilizados para se estimar o componente de variância para a interação reprodutor x rebanho das produções de leite e de gordura e verificar o efeito desta interação sobre a avaliação genética dos reprodutores, por meio de modelos que diferiam na presença e ausência do termo de interação. As produções de leite e de gordura foram ajustadas para duas ordenhas diárias, 305 dias de lactação e idade adulta da vaca. O teste da razão de verossimilhança foi utilizado na verificação da efetividade da inclusão da interação no modelo. As médias das produções de leite e de gordura foram $6085,79 \pm 1629,73 \mathrm{~kg}$ e 225,61 \pm 60,44 kg, respectivamente. A proporção da variância total decorrente da interação reprodutor x rebanho foi $0,4 \%$, para a produção de leite, e $1 \%$, para a produção de gordura. A estimativa de herdabilidade foi 0,38, para a produção de leite, utilizando-se ambos os modelos, e reduziu de 0,40 para 0,39, para a produção de gordura, quando o modelo com interação foi considerado. A função de verossimilhança aumentou significativamente com a inclusão da interação no modelo. A correlação de Spearman foi próxima de um para ambas as características, quando todos os reprodutores foram considerados. Houve redução de 1\% na estimativa de acurácia dos valores genéticos preditos para ambas as características, porém, a correlação de Pearson estimada entre as acurácias obtidas para cada modelo estudado foi próxima à unidade. A interaçãoreprodutor x rebanho não afetou as estimativas de componentes de variâncias genética e residual e a ordem de classificação dos reprodutores para ambas as características.
\end{abstract}

Palavras-chave: acurácia, avaliação genética, bovinos leiteiros, componentes de variância, correlação genética, interação genótipo x ambiente

\section{Effects of Sire $x$ Herd Interaction on Milk and Fat Yields in Brown-Swiss Herds}

\begin{abstract}
Data comprising 4,959 lactations of 2,414 Brown-Swiss cows, daughters of 70 sires, from 51 herds, were used to estimate the variance component for sire $\mathrm{x}$ herd interaction on milk and fat yields and to verify the effect of this interaction on sire genetic evaluation, using the models that differed in the presence or not of the interaction term. The milk and fat yields were adjusted for two milkings, 305 days of lactation and age cow. Likelihood ratio test was used to verify the effectiveness of including a sire $\mathrm{x}$ herd interaction effect in the model. Averages for milk and fat yields were $6085.79 \pm 1629.73 \mathrm{~kg}$ and $225.61 \pm 60.44 \mathrm{~kg}$, respectively. The proportion of the phenotypic variance due to sire $\mathrm{x}$ herd interaction was $0.4 \%$ for milk yield and $1 \%$ for fat yield. The heritability estimate was 0.38 to milk yield, using both models, and decreased from 0.40 to 0.39 for fat yield, when the model with interaction effect was considered. The likelihood function increased significantly with the inclusion of interaction in the model. The Spearman correlation was close to one for both traits, when all sires were considered. There was reduction of $1 \%$ in the estimate of accuracy of the breeding values for both traits, however the Pearson correlation estimated among the accuracy obtained for each model was close to one. No effects of sire $\mathrm{x}$ herd interaction on estimates of genetic and residual variance components, breeding values magnitude and sire order classification, for both traits, were observed.
\end{abstract}

Key Words: accuracy, genetic evaluation, dairy cattle, variance components, genetic correlation, genotype environment interaction

\section{Introdução}

O gado Pardo-Suíço é o segundo maior rebanho especializado em produção de leite, criado no Brasil. No entanto, esta raça é originária de países temperados e seus índices de produção nacional ainda estão aquém da produção verificada nos países de clima temperado. Essa discrepância na produção pode decorrer de diferenças de clima, nutrição, manejo, estado sanitário do rebanho, entre outras, as quais caracterizam a interação genótipo $\times$ ambiente, pois os reprodutores são selecionados em ambientes tempe-

\footnotetext{
${ }^{1}$ Doutoranda em Nutrição e Produção Animal pelo Departamento de Zootecnia da UNESP/FMVZ/Botucatu-SP. Bolsista da Capes. E.mail: mirellasirol@ig.com.br

2 Prof. do Departamento de Zootecnia da UFV, Viçosa-MG.

${ }^{3}$ Prof. do Departamento de Zootecnia da UFV, Viçosa-MG. Bolsista do CNPq.

${ }^{4}$ Profa. Visitante UFV/FAPEMIG.

5 Prof. Adjunto da Universidade Federal Rural da Amazônia.

${ }^{6}$ Doutorando em Zootecnia, UFV, Viçosa-MG. E.mail: fprenno@hotmail.com
} 
rados e suas progênies expressam seus índices produtivos em países de clima tropical.

A existência de interação genótipo $\times$ ambiente, assim como a maneira mais adequada de se tratar este efeito nos modelos de avaliação genética, tem sido estudada por vários autores. Uma das maneiras é a estimação de componentes de variância, comparando-se modelos que incluam ou não o efeito de interação genótipo $\times$ ambiente (Banos \& Shook, 1990; Dimov et al., 1995). Banos \& Shook (1990), utilizando dados de primeira, segunda e terceira lactações de vacas da raça Holandesa, participantes do programa de melhoramento de rebanhos leiteiros de Wisconsin, constataram que a proporção da variância fenotípica decorrente da interação reprodutor $\times$ rebanho foi pequena, evidenciando pouca importância desse efeito na avaliação genética dos reprodutores. Dimov et al. (1995) chegaram à mesma conclusão quando obtiveram a proporção da variância fenotípica resultante da interação reprodutor $\times$ rebanho, ao analisarem dados de primeira, segunda e terceira lactações de vacas da raça Holandesa da Califórnia e Nova York. Porém, Parekh \& Baghel (1991), a partir da análise da produção de leite de vacas criadas em duas regiões da Índia, em primeira lactação, utilizando reprodutores das raças Holandesa, Jersey e Pardo-Suíca, em diferentes cruzamentos, relataram que o componente de interação foi responsável por $15 \%$ da variância total, concluindo que o efeito foi importante.

Costa (1999) relatou, em uma revisão sobre a interação reprodutor $\times$ rebanho em várias raças leiteiras, que este efeito tem representado de 1 a $4 \%$ da variância total da produção de leite.

Outra forma de avaliar o efeito da interação genótipo $\times$ ambiente tem sido a classificação dos animais de acordo com os valores genéticos preditos, por meio dos modelos supracitados, ou seja, com e sem o componente de interação(Mohammad et al., 1982; Basu \& Chattaraji, 1988; Carabaño et al., 1990).

Os resultados encontrados na literatura quanto à mudança na ordem de classificação dos touros em razão da interação genótipo $\times$ ambiente têm sido controversos. Basu \& Chattaraji (1988) afirmaram que ignorar o efeito da interação x reprodutor rebanho pode resultar em mudança na ordem de classificação dos reprodutores, quando esta for baseada na produção de leite de vacas de primeira lactação, provenientes de cruzamentos entre as raças Holandesa e Sahiwal. No entanto, para Mohammad et al.
(1982) e Carabaño et al. (1990), este efeito não alterou significativamente as ordens de classificação dos touros, quando elas foram obtidas com base nos valores genéticos preditos, utilizando-se registros de primeira lactação de vacas holandesas. Os dados utilizados por Mohammad et al. (1982) foram provenientes do programa de melhoramento de rebanhos holandeses de Illinois e os de Carabaño et al. (1990) foram de rebanhos da Califórnia, de Nova York e Wisconsin, nos Estados Unidos.

Cienfuegos-Rivas et al. (1999) e Costa et al. (2000) compararam o desempenho de filhas de touros da raça Holandesa criadas em diferentes países e observaram efeito da interação genótipo $\times$ ambiente, evidenciado tanto pela heterogeneidade de variâncias quanto pelos coeficientes de correlação genética.

Rorato et al. (2000), Torres et al. (2000) e Araújo et al. (2001a) investigaram o mesmo efeito sobre o desempenho produtivo de vacas da raça Holandesa no Brasil; Rorato et al. (2000) dividiram os rebanhos em níveis de produção de leite e Araújo et al. (2001a), em classes de desvio-padrão fenotípico e observaram que os componentes de variância foram heterogêneos e, conseqüentemente, os coeficientes de herdabilidade também o foram.

Considerando-se os resultados contraditórios observados na literatura e a importância da interação genótipo $\times$ ambiente na avaliação genética de reprodutores, objetivou-se estimar os componentes de variância para o efeito da interação reprodutor $x$ rebanho sobre as produções de leite e de gordura, em registros de lactações de vacas da raça Pardo-Suíça e verificar a importância do efeito dessa interação na avaliação genética dos reprodutores.

\section{Material e Métodos}

Foram utilizados registros de 4.959 lactações provenientes de 2.414 vacas, filhas de 70 reprodutores, distribuídas em 51 rebanhos, com parições no período de 1985 a 1998. Os registros foram obtidos junto ao Controle Leiteiro da Associação Brasileira dos Criadores de Gado Pardo-Suíço. As lactações foram ajustadas para duas ordenhas diárias, 305 dias de lactação e para produção na idade adulta da vaca, de acordo com os ajustes citados por Rennó (2001). Registros de lactações incompletas e encerradas por causas anormais de secagem, lactações inferiores a 150 e superiores a 365 dias, registros de produção de leite inferiores a 1.000 e superiores a $15.000 \mathrm{~kg}$ e 
idade da vaca ao parto menores que 18 e maiores 197 meses foram eliminados. Os meses de parto das vacas foram agrupados em quatro estações: 1) janeiro a março, 2) abril a junho, 3) julho a setembro e 4) outubro a dezembro. As estações 1 e 4 corresponderam aos meses de maior incidência de chuvas, enquanto as estações 2 e 3, aos meses de menor incidência, uma vez que os dados analisados, em sua maioria, foram provenientes de rebanhos localizados principalmente nas regiões Sudeste e Sul do país.

Para que fosse possível investigar o efeito da interação reprodutor $\times$ rebanho foi exigido que cada reprodutor tivesse, no mínimo, quatro filhas, distribuídas em pelo menos dois rebanhos diferentes, de modo que cada rebanho deveria conter ao menos duas filhas. Quanto às informações de ascendência e origem, as vacas foram classificadas em três grupos genéticos, ou seja, vacas Pardo-Suíças puras de origem, puras por cruzamento e puras de origem importada.

Os componentes de (co)variância foram estimados pelo método da máxima verossimilhança restrita, utilizando-se o programa MTDFREML ("Multiple Trait Derivative Free Restricted Maximum Likelihood”), descrito por Boldman et al. (1995). O critério de convergência adotado foi a variância dos valores do simplex igual a $10^{-9}$.

Dois modelos multicaracterísticos para produção de leite e gordura, os quais incluíam os efeitos fixos de rebanho, ano-estação de parto, grupo genético do animal, efeito aleatório de animal e de ambiente permanente, foram considerados para se estimar os componentes de variância. A diferença estava na inclusão do efeito aleatório de interação reprodutor $\times$ rebanho em um dos modelos.

Com base nos valores genéticos preditos, para ambos os modelos, foram calculadas as correlações de Spearman, considerando-se todos os reprodutores e, posteriormente, considerando-se 10, 20, 30, 40 e $50 \%$ dos 70 touros analisados, para se verificar possíveis alterações na classificação dos reprodutores, de acordo com cada modelo.

A fim de se verificar o efeito da inclusão do termo referente à interação reprodutor $\times$ rebanho na avaliação genética dos reprodutores, foram estimadas as acurácias (Rti) dos valores genéticos, para ambos os modelos, da seguinte forma: $\mathrm{Rti}=\sqrt{1-\frac{\mathrm{PEV}}{\sigma_{a}^{2}}}$, em que PEV é a variância do erro de predição e $\sigma_{\mathrm{a}}^{2}$, a variância genética aditiva de cada característica.
Para se comprovar que, ao ignorar o efeito de interação na avaliação genética dos reprodutores, poderia estar ocorrendo superestimação dos valores genéticos preditos, estimou-se a correlação de Pearson entre os valores de acurácia dos valores genéticos preditos obtidos para os modelos, sem e com o efeito de interação reprodutor $\times$ rebanho.

A correlação de Spearman para a ordem dos valores genéticos preditos e de Pearson para a acurácia, e toda a editoração e montagem de arquivos necessários para as análises foram feitas pelo sistema SAS ${ }^{\circledR}$ (1990).

Também foi realizado o teste da razão de verossimilhança de modelos seqüencialmente reduzidos (Rao, 1973), para se determinar a importância da inclusão do efeito de interação reprodutor $\times$ rebanho no modelo de avaliação genética de reprodutores.

\section{Resultados e Discussão}

As médias observadas para as produções de leite e de gordura e os desvios-padrão de cada característica foram 6085,79 \pm 1629,73 kg e 225,607 \pm 60,4683 kg, respectivamente, que foram superiores às encontradas em outros trabalhos realizados com animais da raça Pardo-Suíça no Brasil. Araújo et al. (2002), trabalhando com 6.842 lactações de 3.274 vacas da raça Pardo-Suíça, distribuídas em 100 rebanhos e Rennó et al. (2002), com 11.189 lactações de 5.382 vacas da mesma raça, distribuídas em 201 rebanhos, encontraram médias de produção de leite e desviospadrão de 5.941,43 \pm 1.661,66 kg e 5.791,50 $\pm 1.211,58 \mathrm{~kg}$ e, para a produção de gordura, 221,28 $\pm 61,13 \mathrm{~kg}$ e $217,25 \pm 47,36 \mathrm{~kg}$, respectivamente. Em razão das diferenças nas restrições impostas ao arquivo de dados para que se pudesse avaliar o efeito da interação reprodutor $\times$ rebanho, o número de reprodutores, de lactações e de rebanhos utilizados neste estudo foi menor que aquele dos estudos supracitados, resultando em médias inferiores.

Os componentes de (co)variância estimados pelos modelos sem e com o termo de interação reprodutor $\times$ rebanho podem ser observados na Tabela 1 .

Houve redução nas estimativas dos componentes de (co)variância, para ambas as características, quando o efeito de interação reprodutor $\times$ rebanho foi incluído no modelo, exceto no componente de variância do efeito de ambiente permanente da produção de gordura do leite, que foi quase nulo, em ambos os modelos, e nos componentes de (co)variância residuais, que permaneceram praticamente inalterados. 
Tabela 1 - Componentes de (co)variância genética aditiva, de ambiente permanente, de interação reprodutor $x$ rebanho e residual, para as produções de leite e de gordura

Table 1 - Additive genetic components of (co)variance, permanent environmental, sire $x$ herd interaction and error variances for milk and fat yields

\begin{tabular}{|c|c|c|c|c|}
\hline \multirow[b]{2}{*}{$\begin{array}{l}\text { Característica* } \\
\text { Trait }^{*}\end{array}$} & \multicolumn{2}{|c|}{$\begin{array}{l}\text { Modelo sem interação } \\
\text { Model without interaction }\end{array}$} & \multicolumn{2}{|c|}{$\begin{array}{l}\text { Modelo com interação } \\
\text { Model with interaction }\end{array}$} \\
\hline & $\begin{array}{l}\text { PL } \\
M Y\end{array}$ & $\begin{array}{l}\text { PG } \\
F Y\end{array}$ & $\begin{array}{l}\text { PL } \\
M Y\end{array}$ & $\begin{array}{l}\text { PG } \\
F Y\end{array}$ \\
\hline \multirow{3}{*}{$\begin{array}{l}\mathrm{PL}^{1}(M Y) \\
\mathrm{PG}^{2}(F Y)\end{array}$} & \multicolumn{4}{|c|}{$\begin{array}{l}\text { (Co)variância genética aditiva }\left(\mathrm{kg}^{2}\right) \\
\text { Genetic additive (co)variance }\left(\mathrm{kg}^{2}\right)\end{array}$} \\
\hline & 636743,850 & $\begin{array}{l}23350,1979 \\
992,6297\end{array}$ & 636168,3259 & $\begin{array}{c}23196,1389 \\
954,4900\end{array}$ \\
\hline & \multicolumn{4}{|c|}{$\begin{array}{l}\text { Variância de ambiente permanente }\left(\mathrm{kg}^{2}\right) \\
\text { Permanent environmental variance }\left(\mathrm{kg}^{2}\right)\end{array}$} \\
\hline \multirow[t]{2}{*}{$\begin{array}{l}\text { PL }(M Y) \\
\operatorname{PG}(F Y)\end{array}$} & 22018,2641 & 0,0008 & 16653,6189 & 0,0056 \\
\hline & \multicolumn{4}{|c|}{$\begin{array}{l}\text { Variância de interação reprodutor x rebanho }\left(\mathrm{kg}^{2}\right) \\
\text { Sire } \mathrm{x} \text { herd interaction variance }\left(\mathrm{kg}^{2}\right)\end{array}$} \\
\hline $\begin{array}{l}\text { PL }(M Y) \\
\text { PG }(F Y)\end{array}$ & & $\begin{array}{l}\text { (Co)variân } \\
\text { Error (cc }\end{array}$ & $\begin{array}{l}\quad 6887,25 \\
\text { dual }\left(\mathrm{kg}^{2}\right) \\
\text { ce }\left(\mathrm{kg}^{2}\right)\end{array}$ & 27,8765 \\
\hline $\begin{array}{l}\text { PL }(M Y) \\
\text { PG }(F Y)\end{array}$ & 1000842,2419 & $\begin{array}{c}33957,6426 \\
1498,1064\end{array}$ & 1000070,8727 & $\begin{array}{r}33922,9885 \\
1495,9962\end{array}$ \\
\hline
\end{tabular}

${ }^{1} \mathrm{PL}=$ produção de leite, em kg; ${ }^{2} \mathrm{PG}$ = produção de gordura, em kg.

${ }^{1} \mathrm{MY}=$ milk yield, in $\mathrm{kg} ;{ }^{2} \mathrm{FY}=$ fat yield, in $\mathrm{kg}$.

* PL e PG foram ajustadas às duas ordenhas diárias, aos 305 dias de lactação e à idade adulta.

${ }^{*} \mathrm{MY}$ and FY were adjusted for 305-days, $2 x$ and mature equivalent to age cow.

Reduções nos componentes de variância, ao comparar modelos que diferiam na ausência e presença do efeito da interação reprodutor $\times$ rebanho, foram relatadas por Tong et al. (1977), Meyer (1987) e Banos \& Shook (1990), para a raça Holandesa.

Araújo et al. (2001a) registraram reduções de 4 e $10 \%$, respectivamente, nos componentes de variância genética aditiva e de ambiente permanente da produção de leite, mas não encontraram alteração no componente de variância residual quando o modelo incluiu o termo de interação reprodutor $\times$ rebanho, na avaliação genética de reprodutores holandeses no Brasil.

Van Tassel \& Berger (1994), por meio de simulação, investigaram as conseqüências de serem ignorados o termo de interação reprodutor $\times$ rebanho e o parentesco dos reprodutores na estimação de componentes de variância e observaram superestimação do componente de variância do efeito de reprodutor quando se desconsiderou a interação reprodutor $x$ rebanho, viés que foi dependente dos níveis de interação simulados nos dados. Discutiram também que, quando o parentesco entre os reprodutores foi ignorado, as variâncias decorrentes do efeito de reprodutor e a interação reprodutor $\times$ rebanho tenderam a serem subestimadas, à medida que os reprodutores apresentavam parentesco mais próximo.

A informação proveniente da matriz de numeradores dos coeficientes de parentesco foi ignorada, o que poderia causar algum viés nessa estimativa. Porém, o componente de interação reprodutor $\times$ rebanho foi baixo e, segundo Araújo et al. (2001b), a perda de informação é muito pequena ao desconsiderar a informação de parentesco entre reprodutores, quando o nível de interação também é baixo.

Os valores de herdabilidades e proporções da variância fenotípica, decorrentes de efeitos de ambiente permanente, de interações reprodutor x rebanho e residual, estimados para os modelos sem e com interação, para ambas as características, encontram-se na Tabela 2.

As estimativas de componentes de variâncias da produção de leite para o efeito genético aditivo e para o efeito de ambiente temporário foram muito próximas ao se incluir e não incluir a interação reprodutor 
Tabela 2 - Estimativas de herdabilidade $\left(\mathrm{h}^{2}\right)$ e proporções da variância total, decorrentes de efeitos de ambiente permanente $\left(C^{1}\right)$, de interações reprodutor $x$ rebanho $\left(C^{2}\right)$ e residual $(e)$, estimados para os modelos sem e com efeito de interação reprodutor $x$ rebanho, para a produção de leite e de gordura

Table 2 - Heritability $\left(h^{2}\right)$ and proportion of total variance due to permanent environmental effect $\left(C^{1}\right)$, sire $x$ herd interaction $\left(C^{2}\right)$ and error (e) estimated by the models without and with effect of sire $x$ herd interaction for milk and fat yields

\begin{tabular}{|c|c|c|c|c|}
\hline & \multicolumn{4}{|c|}{$\begin{array}{c}\text { Característica } \\
\text { Trait }\end{array}$} \\
\hline & \multicolumn{2}{|c|}{$\begin{array}{c}\text { Produção de leite } \\
\text { Milkyield }\end{array}$} & \multicolumn{2}{|c|}{$\begin{array}{c}\text { Produção de gordura } \\
\text { Fat yield }\end{array}$} \\
\hline & $\mathrm{SI}^{1}$ & $\mathrm{CI}^{2}$ & SI & $\mathrm{CI}$ \\
\hline$h^{2}$ & 0,38 & 0,38 & 0,40 & 0,39 \\
\hline$C^{1}$ & 0,0132 & 0,0100 & 0,0000 & 0,0000 \\
\hline$C^{2}$ & - & 0,0041 & - & 0,0112 \\
\hline e & 0,60 & 0,60 & 0,60 & 0,60 \\
\hline
\end{tabular}

${ }^{1} \mathrm{SI}=$ modelo sem interação (model without interaction).

${ }^{2} \mathrm{Cl}=$ modelo com interação (model with interaction).

$\times$ rebanho no modelo. Conseqüentemente, as estimativas de herdabilidade para a produção de leite foram as mesmas, em ambos os modelos.

Para a produção de gordura, a estimativa de herdabilidade reduziu de 0,40 para 0,39. A correlação genética entre as produções de leite e de gordura aumentou de 0,93 para 0,94, quando o efeito de interação reprodutor $\times$ rebanho foi considerado no modelo. Esta pequena redução na estimativa de herdabilidade para a produção de gordura e o aumento na correlação entre a produção de leite e de gordura podem ser explicados pela maior redução do componente de variância genética aditiva estimado para a produção de gordura.

As estimativas de herdabilidade e correlações genéticas foram superiores às encontradas por Costa et al. (2000), que, utilizando registros de produções de leite e de gordura de vacas da raça Holandesa, avaliaram a efeito da interação genótipo $\times$ ambiente entre países e encontraram estimativas de herdabilidade para a produção de leite e de gordura de 0,25 e 0,22, no Brasil, e de 0,34 e 0,35, nos Estados Unidos, e de correlação genética entre as características de 0,79, no Brasil, e de 0,62, nos Estados Unidos. Uma das razões das maiores estimativas, tanto de herdabilidade como de correlações genéticas, podem ser explicadas pela maior variabilidade genética nos rebanhos, visto que o processo de seleção artificial na raça Pardo-Suíça brasileira é recente, quando comparado aos rebanhos de países com maior tradição em pecuária leiteira.

Os resultados deste estudo também foram superiores aos de Araújo et al. (2001b) que, ao verificarem o efeito da interação reprodutor $\times$ rebanho sobre a acurácia da avaliação genética de reprodutores da raça Holandesa no Brasil, encontraram valores de herdabilidade e seus respectivos desvios-padrão de $0,247 \pm 0,021$ e $0,237 \pm 0,021$, para a produção de leite, utilizando modelos com e sem interação reprodutor $\times$ rebanho, respectivamente. Esses autores relataram que, considerando-se os desvios-padrão, os valores de herdabilidade não variaram para a produção de leite entre os modelos e atribuíram o fato à pequena diferença observada nas estimativas de componentes de variância entre eles, como verificado neste estudo.

A proporção da variância total resultante da interação reprodutor $\times$ rebanho foi $0,4 \%$, para a produção de leite, e em torno de 1\%; ou seja, foram inferiores às citadas por Meyer (1987), de 2,22 a 3\%, para produção de leite, e de 2,10 a 3,18\%, para produção de gordura.

Rorato et al. (2000) encontraram valores iguais a 7, 4 e 4\% em razão da interação reprodutor $\times$ anoépoca de parto, respectivamente, para os níveis baixo, médio e alto de produção de leite, em rebanhos da raça Holandesa, no Brasil. Araújo et al. (2001b) relataram que o componente de variância resultante da interação reprodutor $\times$ rebanho representou 3,4\% da variância total da produção de leite de vacas Holandesas.

A proporção observada foi muito baixa, tanto para a produção de leite como para a de gordura, e apresenta pouca importância, pois, segundo Kelleher (1967), o componente de interação só seria importante quando fosse responsável por $10 \%$ ou mais da variância total da característica.

Apesar de os componentes de variância e as herdabilidades terem sido muito pouco alterados e a proporção da variância fenotípica decorrente da interação reprodutor $\times$ rebanho ser muito baixa, observou-se aumento significativo $(\mathrm{P}<0,01)$ da função de verossimilhança, ao se considerar a interação no modelo, de acordo com o teste da razão da verossimilhança, para modelos seqüencialmente reduzidos, cujo valor foi 36,26, o que indicou superioridade do modelo que considerou a interação. O mesmo foi relatado por 
Meyer (1987) quando comparou modelos que incluíam o efeito de interação reprodutor $\times$ rebanho e reprodutor x rebanho-ano-estação com o modelo que desconsiderava essas interações. Araújo et al. (2001a,b) também verificaram aumento significativo da função de verossimilhança, ao avaliarem a importância da inclusão do efeito de interação reprodutor $\times$ rebanho na produção de leite e na acurácia da avaliação genética de reprodutores, respectivamente, utilizando registros de vacas Holandesas.

As médias dos valores genéticos preditos para os reprodutores, os desvios-padrão, valores máximos e mínimos e as amplitudes obtidas para as produções de leite e de gordura, sob os modelos sem e com interação reprodutor $\times$ rebanho podem ser observadas na Tabela 3.

A média dos valores genéticos preditos aumentou quando a interação foi considerada, mas a amplitude permaneceu quase inalterada para ambas as características, indicando que não houve mudanças significativas nas magnitudes dos valores genéticos quando o efeito da interação reprodutor $\times$ rebanho foi considerado no modelo.

Tabela 3 - Médias, desvios-padrão, valores máximos e mínimos e amplitudes dos valores genéticos preditos para os reprodutores, obtidos para as produções de leite e de gordura, utilizando-se os modelos sem e com interação reprodutor $\mathrm{x}$ rebanho

Table 3 - Averages, standard deviation, maximum and minimum values and amplitude of estimated breeding values for sires, obtained for milk and fat yields, using the models without and with sire $x$ herd interaction

\begin{tabular}{|c|c|c|c|c|}
\hline & \multicolumn{4}{|c|}{$\begin{array}{l}\text { Valores genéticos preditos } \\
\text { Estimated breeding values }\end{array}$} \\
\hline & \multicolumn{2}{|c|}{$\begin{array}{c}\text { Produção } \\
\text { de leite } \\
\text { Milkyield }\end{array}$} & \multicolumn{2}{|c|}{$\begin{array}{l}\text { Produção } \\
\text { de gordura } \\
\text { Fat yield }\end{array}$} \\
\hline & $\mathrm{SI}^{1}$ & $\mathrm{CI}^{2}$ & SI & $\mathrm{CI}$ \\
\hline $\begin{array}{l}\text { Média }(\mathrm{kg}) \\
\text { Average }\end{array}$ & 41,5056 & 41,8787 & 0,6597 & 0,7882 \\
\hline $\begin{array}{l}\text { Desvio-padrão (kg) } \\
\text { Standard deviation }\end{array}$ & 541,9 & 537,0 & 21,2810 & 20,8127 \\
\hline $\begin{array}{l}\text { Mínimo (kg) } \\
\text { Minimum }\end{array}$ & $-1960,5$ & $-1920,3$ & $-72,4360$ & $-72,3720$ \\
\hline $\begin{array}{l}\text { Máximo }(\mathrm{kg}) \\
\text { Maximum }\end{array}$ & 968,7 & 986,3 & 44,4550 & 42,8060 \\
\hline $\begin{array}{l}\text { Amplitude (kg) } \\
\text { Amplitude }\end{array}$ & 2929,2 & 2906,6 & 116,891 & 115,178 \\
\hline
\end{tabular}

$1 \mathrm{SI}=$ modelo sem interação (model without interaction).

$2 \mathrm{Cl}=$ modelo com interação (model with interaction).
Basu \& Chattaraji (1988) também verificaram aumento no valor genético predito dos touros, para a produção de leite, ao utilizarem registros de primeira lactação de vacas resultantes do cruzamento entre as raças Holandesas e Sahiwal, por meio de modelos que diferiam na ausência e presença do termo de interação reprodutor $\times$ rebanho. Os autores sugeriram que, ao ignorar a interação, os reprodutores avaliados por meio de filhas mestiças poderiam ter suas ordens de classificação alteradas.

Apesar do pequeno aumento verificado na média dos valores genéticos preditos, tanto para a produção de leite como para a de gordura, a ordem de classificação dos touros não foi alterada de maneira significativa, como pode ser observado na Tabela 4, onde são apresentadas as correlações de Spearman entre os valores genéticos preditos para as produções de leite e de gordura, utilizando-se ambos os modelos.

As correlações de Spearman acima de 0,99 indicaram que o ordenamento dos reprodutores, com base nos valores genéticos preditos, na presença do efeito de interação reprodutor $\times$ rebanho, não foi alterado. Mohammad et al. (1982) observaram que ignorar o efeito de interação na estimativa dos valores genéticos não causaria maiores alterações na classificação dos reprodutores, uma vez que houve correlações altas $(0,99)$. Também Araújo et al. (2001a) e Araújo et al. (2001b), avaliando o efeito da interação reprodutor $\times$ rebanho na avaliação genética da raça Holandesa, encontraram correlações de Spearman próximas à unidade, indicando que os reprodutores seriam classificados de maneira similar ao se utilizar o modelo com ou sem interação.

Por outro lado, vários autores avaliaram o efeito da interação genótipo $\times$ ambiente na avaliação genética de reprodutores entre países (Abubakar et al., 1987; Stanton et al., 1991) e obtiveram resultados contraditórios.

Abubakar et al. (1987) estudaram reprodutores da raça Holandesa com base nas informações de filhas criadas no México e Colômbia e estimaram correlação genética entre a ordem de classificação dos reprodutores igual a 0,26. Stanton et al. (1991), por sua vez, utilizando registros de primeira lactação de vacas Holandesas da Colômbia, do México, de Porto Rico e dos Estados Unidos, concluíram que as estimativas de correlação entre todos os países da América Latina e dos Estados Unidos foram suficientemente grandes para sugerir que o ordenamento dos reprodutores não seria alterado em cada ambiente. 
Tabela 4 - Correlações de Spearman entre as ordens de classificação dos reprodutores, com base nos valores genéticos preditos para as produções de leite e de gordura, utilizando ambos os modelos sem e com interação reprodutor $x$ rebanho

Table 4 - Spearman correlations among the rank of the sires based on estimated breeding values for milk and fat yields, using both models without and with sire $x$ herd interaction

\begin{tabular}{lccc}
\hline & VGPLCI & VGPGSI & VGPGCI $^{4}$ \\
& $E B V M I$ & $E B V F$ & $E B V F I^{4}$ \\
\hline VGPLSI $^{1}(E B V M)$ & 0,9993 & 0,8993 & 0,9243 \\
VGPLCI $^{2}(E B V M I)$ & & 0,9051 & 0,9281 \\
VGPGSI $^{3}(E B V F)$ & & & 0,9935 \\
\hline
\end{tabular}

${ }^{1}$ VGPLSI = Valor genético predito para a produção de leite, utilizando o modelo sem interação; ${ }^{2} \mathrm{VGPLCl}=$ Valor genético predito para a produção de leite, utilizando o modelo com interação; ${ }^{3}$ VGPGSI = Valor genético predito para a produção de gordura, utilizando o modelo sem interação; ${ }^{4}$ VGPGCI = Valor genético predito para a produção de gordura, utilizando o modelo com interação.

${ }^{1} E B V M=$ Estimated breeding value for milk yield, using the model without the effect of sire $x$ herd interaction; ${ }^{2} E B V M I=$ Estimated breeding value for milkyield, using the model with the effect of sire $x$ herd interaction; ${ }^{3} E B V F$ = Estimated breeding value for fat yield, using the model without the effect of sire $x$ herd interaction; ${ }^{4} E B V F I=$ Estimated breeding value for fat yield, using the model with effect of sire $x$ herd interaction.

Essa diferença na estimativa das correlações entre países comparadas com as estimativas dentro do Brasil, como neste trabalho, deve ocorrer, provavelmente, pela maior influência de fatores ambientais mais contrastantes entre os países, como clima, manejo, nutrição, entre outros.

Ao se considerarem 10, 20, 30, 40 e $50 \%$ dos reprodutores, observou-se que a correlação de Spearman estimada, dentro de cada característica, entre valores genéticos preditos nos dois modelos, elevou com o aumento do número de reprodutores considerados e que foi acima de 0,89, tanto para produção de leite como para a de gordura. Quando se trabalha com amostra selecionada, espera-se que essa correlação seja reduzida, pois se restringe o número de animais avaliados. Mesmo considerando somente $10 \%$ dos melhores reprodutores, a correlação se manteve alta, podendo-se inferir que o efeito da interação reprodutor $\times$ rebanho não alterou significativamente a ordem de classificação dos touros.

Quanto às acurácias dos valores genéticos, verificou-se que aquelas obtidas com base nos valores genéticos preditos para a produção de leite quase não foram alteradas, com a inclusão do efeito de interação reprodutor $\times$ rebanho, ou seja, somente 13 dos 70 touros tiveram o valor de acurácia reduzido em $1 \%$.
Essa pequena variação pode ser explicada pela redução, também pequena, na variância genética aditiva quando o efeito de interação foi considerado. Conseqüentemente, a estimativa de acurácia também variou muito pouco, resultando em valores semelhantes para ambos os modelos utilizados.

Para a produção de gordura, a redução na acurácia dos valores genéticos preditos também foi de $1 \%$, porém, para a maioria dos reprodutores. Observou-se que, ao ignorar a interação reprodutor $\times$ rebanho, a amplitude dos valores genéticos preditos aumentou e, apesar deste aumento ter sido pequeno, houve aumento no componente de variância genética aditiva. Como conseqüência, foram obtidos menores valores para a variância do erro de predição, causando ligeira superestimação da acurácia da maioria dos valores genéticos preditos para a produção de gordura.

O aumento na amplitude dos valores genéticos foi menor para a produção de leite que para a produção de gordura, o que resultou em um aumento também menor no componente de variância. A ocorrência destes fatos causou superestimação da acurácia dos valores genéticos preditos para a produção de leite somente para alguns reprodutores.

A inclusão da interação reprodutor $\times$ rebanho no modelo de avaliação genética é mais eficiente em situações em que reprodutores apresentam suas proles em poucos rebanhos. Assim, pode haver superestimação dos valores genéticos preditos para reprodutores cujas progênies estão localizadas em rebanhos com maior produtividade, ao passo que, para aqueles reprodutores cujas proles se concentram em rebanhos com menor produtividade, espera-se subestimação na predição de seus valores genéticos.

A correlação de Pearson estimada entre as acurácias obtidas para os valores genéticos preditos, utilizando-se os modelos sem e com interação, foi próxima à unidade, indicando que não houve mudança na acurácia e, conseqüentemente, superestimação significativa dos valores genéticos quando se ignorou o efeito de interação.

\section{Conclusões}

A interação reprodutor $\times$ rebanho não afetou as estimativas de componentes de variância e os parâmetros genéticos das produções de leite e de gordura, assim como a ordem de classificação dos reprodutores da raça Pardo-Suíça. 


\section{Agradecimento}

À Universidade Federal de Viçosa, pela oportunidade; ao CNPq, pela concessão de bolsas de pesquisa; e à Associação Nacional de Criadores de Gado Pardo-Suíço, pelo fornecimento dos dados.

\section{Literatura Citada}

ABUBAKAR, B.Y.; Mc DOWELL, R.E.; Van VLECK, L.D. Interaction of genotype and environment for breeding efficiency and milk production of Holsteins in Mexico and Colombia. Tropical Agriculture, v.64, n.1, p.17-24, 1987.

ARAÚJO, C.V.; TORRES, R.A.; COSTA, N.C. et al. Interação reprodutor x rebanho na produção de leite da raça Holandesa no Brasil. Revista Brasileira de Zootecnia, v.30, n.3, p.992-999, 2001a (suplemento 1).

ARAÚJO, C.V.; TORRES, R.A.; COSTA, N.C. et al. Efeito da interação reprodutor $\mathrm{x}$ rebanho na produção de leite da raça Holandesa no Brasil. Revista Brasileira de Zootecnia, v.30, n.3, p.1000-1006, 2001b (suplemento 1).

ARAÚJO, C.V.; TORRES, R.A.; RENNÓ, F.P. et al. Heterogeneidade de variância na avaliação genética de reprodutores da raça Pardo-Suíça no Brasil. Revista Brasileira de Zootecnia, v.31, n.3, p.1343-1349, 2002.

BANOS, G.; SHOOK, G.E. Genotype by environment interaction and genetic correlations among parities for somatic cell count and milk yield. Journal of Dairy Science, v.73, n.9, p.2563-2573, 1990.

BASU, S.B.; CHATTARAJI, J. Genotype-environment interaction in the evaluation of sires of crossbred cows. Indian Journal of Animal Science, v.58, n.7, p.808-811, 1988.

BOLDMAN, K.G.; KRIESE, L.A.; Van VLECK, L.D. et al. A manual for use of MTDFREML: a set of programs to obtain estimates of variances and covariances (DRAFT). Lincoln: Department of Agriculture/Agriculture Research Service, 1995. 125p.

CARABAÑO, M.J.; Van VLECK, L.D.; WIGGANS, G.R. et al. Estimation of genetic parameters for milk and fat yields of dairy catlle in Spain and the United States. Journal of Dairy Science, v.72, n.11, p.3013-3022, 1990.

CIENFUEGOS-RIVAS, E.G.; OLTENACU, P.A.; BLAKE, R.W. et al. Interaction between milk yeild of holstein cows in Mexico and the United States. Journal of Dairy Science, v.82, n.10, p.2218-2223, 1999.

COSTA, C.N. An investigation into heterogeneity of variance for milk and fat yields of holstein cows in brasilian herd environments. Genetics and Molecular Biology, v.22, n.3, p.375-381, 1999.

COSTA, C.N.; BLAKE, R.W.; POLLAK, E.J. et al. Genetic analysis of Holstein cattle populations in Brazil and United States. Journal Dairy Science, v.83, n.12, p.2963-2974, 2000.

DIMOV, G.; ALBUQUERQUE, L.G.; KEOWN, J.F. et al. Variance of interaction effects of sire and herd for yield traits of Holsteins in California, New York, and Pennsylvania with an animal model. Journal of Dairy Science, v.78, n.4, p.939-946, 1995.
KELLEHER, D.J.; FREEMAN, A.E.; LUSH, J.L. Importance of bull $\mathrm{x}$ herd-year-season interaction in milk production. Journal of Dairy Science, v.50, n.10, p.1703-1707, 1967.

MEYER, K. Estimates of variance due to sire $\mathrm{x}$ herd interactions and environmental covariances between paternal half-sibs for first lactation dairy production. Livestock Production Animal Science, v.17, n.1, p.95-115, 1987.

MOHAMMAD, W.A.; LEE, A.J.; GROSSMAN, M. Genotypeenvironment interaction in sire evaluation. Journal of Dairy Science, v.65, n.5, p.857-860, 1982.

PAREKH, H.K.B.; BAGHEL, A.S. Genotype x environment interaction for milk production using crossbred progenies in India: different approaches for its estimation. Indian Journal of Dairy Science, v.44, n.7, p.406-411, 1991.

RAO, C.R. Linear statistical inference and its aplications. 2.ed. New York: John Wiley \& Sons, 1973. 552p.

RENNÓ, F.P. Aspectos produtivos da raça Pardo-Suíça no Brasil. Viçosa, MG: Universidade Federal de Viçosa, 2001. 100p. Dissertação (Mestrado em Zootecnia) - Universidade Federal de Viçosa, 2001.

RENNÓ, F.P.; PEREIRA, J.C.; ARAÚJO, C.V. et al. Aspectos produtivos da raça Pardo-Suíça no Brasil. Fatores de ajustamento, produção de leite e de gordura, e parâmetros genéticos. Revista Brasileira de Zootecnia, v.31, n.5, p.2043-2054, 2002.

RORATO, P.R.N.; Van VLECK, D.; VERNEQUE, R.S. et al. Interação genótipo-ambiente para a produção de leite em rebanhos da raça Holandesa no Brasil. 2.Uso de um modelo animal. Revista Brasileira de Zootecnia, v.29, n.6, p.20302035, 2000 (Suplemento 1).

STANTON, T.L.; BLAKE, R.W.; QUAAS, R.L. et al. Genotype by enviroment interaction for Holstein milk yield in Colombia, México and Porto Rico. Journal of Dairy Science, v.74, n.5, p.1700-1714, 1991.

STATISTICAL ANALYSES SYSTEM - SAS. SAS user's guide: statistics. version 6, 4.ed. Cary: 1990. 1686p.

TONG, A.K.W.; KENNEDY, B.W.; MOXLEY, J.E. Sire by herd interactions for milk yield and composition traits. Canadian Journal of Animal Science, v.57, n.3, p.383388, 1977.

TORRES, A.R.; BERGMANN, J.A.G.; COSTA, C.N. et al. Heterogeneidade de variancia e avaliação genética de bovinos da raça Holandesa no Brasil. Revista Brasileira de Zootecnia, v.29, n.4, p.1050-1059, 2000.

Van TASELL, C.P; BERGER, P.J Consideration of sire relationships for estimation of variance components with interaction of herd and sire. Jounal of Dairy Science, v.77, n.1, p.313-324, 1994. 